Please do not remove this page

RMIT

UNIVERSITY

\title{
Experiments and parametric studies on 3D metallic auxetic metamaterials with tuneable mechanical properties
}

Ren, Xin; Shen, Jianhu; Ghaedizadeh, Arash; Tian, Hongqi; Xie, Yi Min

https://researchrepository.rmit.edu.au/esploro/outputs/9921862591501341/filesAndLinks?institution=61RMIT_INST\&index=null

Ren, X., Shen, J., Ghaedizadeh, A., Tian, H., \& Xie, Y. M. (2015). Experiments and parametric studies on 3D metallic auxetic metamaterials with tuneable mechanical properties. Smart Materials and Structures, 24(9), 1-15. https://doi.org/10.1088/0964-1726/24/9/095016

Document Version: Accepted Manuscript

Published Version: https://doi.org/10.1088/0964-1726/24/9/095016

Repository homepage: https://researchrepository.rmit.edu.au

(c) 2015 IOP Publishing Ltd

Downloaded On 2023/04/26 18:58:50 +1000 
Thank you for downloading this document from the RMIT Research Repository.

The RMIT Research Repository is an open access database showcasing the research outputs of RMIT University researchers.

RMIT Research Repository: http://researchbank.rmit.edu.au/

\section{Citation:}

Ren, X, Shen, J, Ghaedizadeh, A, Tian, H and Xie, Y 2015, 'Experiments and parametric studies on 3D metallic auxetic metamaterials with tuneable mechanical properties', Smart Materials and Structures, vol. 24, no. 9, 095016, pp. 1-15.

See this record in the RMIT Research Repository at:

https://researchbank.rmit.edu.au/view/rmit:33389

Version: Accepted Manuscript

Copyright Statement:

๑) 2015 IOP Publishing Ltd

Link to Published Version:

http://dx.doi.org/10.1088/0964-1726/24/9/095016 


\title{
Experiments and parametric studies on 3D metallic auxetic metamaterials with tuneable mechanical properties
}

\author{
Xin Ren ${ }^{1,2}$, Jianhu Shen ${ }^{1}$, Arash Ghaedizadeh ${ }^{1}$,Hongqi Tian ${ }^{2}$, and Yi Min Xie ${ }^{1 *}$ \\ ${ }^{1}$ Centre for Innovative Structures and Materials, School of Civil, Environmental and Chemical Engineering, \\ RMIT University, GPO Box 2476, Melbourne 3001, Australia \\ ${ }^{2}$ Key Laboratory of Traffic Safety on Track, School of Traffic \& Transportation Engineering, \\ Central South University, Changsha 410075, Hunan Province, China
}

\section{Abstract:}

Auxetic metamaterials are synthetic materials with microstructures engineered to achieve negative Poisson's ratios. There has been intense interest in auxetic metamaterials due to their unusual properties and many potential applications. However, most of the previous research was focused on auxetic behaviour of elastomers under elastic deformations. Inspired by our recent finding of the loss of auxetic behaviour in auxetic metamaterials when the base material was changed from elastomer to metal, we have carried out a series of experiments and finite element simulations to investigate the mechanism behind this phenomenon. Employing an improved methodology of generating buckling-induced auxetic metamaterials, several samples of metallic auxetic metamaterials have been fabricated using 3D printing technique. Furthermore, parametric studies have been performed through experimentally validated finite element models to explore the auxetic performance of the designed metamaterials. It is found that the auxetic performance can be tuned by the geometry of microstructures; and the strength and stiffness can be tuned by the plasticity of the base material while maintaining the auxetic performance.

Keywords: Auxetic metamaterial, Metallic material, Plastic deformation, Buckling-induced

\section{Highlights:}

1) Comprehensive parametric studies on a newly developed $3 D$ metallic auxetic metamaterial have been carried.

2) The computational models have been validated by experimental results.

3) Special features of auxetic behaviour related to metallic material plasticity have been identified and investigated.

4) Key parameters for individually tuning the properties of metallic auxetic metamaterials have been identified.

\section{Introduction}


Designing the periodic microstructures of metamaterials allows us to achieve unusual and sometimes even unprecedented effective material properties, which are gained predominantly from shape of microstructure rather than their chemical composition [1-3]. The cellular material with a negative Poisson's ratio (NPR) is the most studied category of mechanical metamaterials. Under uniaxial compression, such materials contract (rather than expand) transversely. They are also known as "auxetic materials" by Evans [4].

An increasing interest in the development of auxetic materials has been aroused because of their many potential applications in various fields, such as prostheses [5], piezoelectric sensors with desired performance [6], energy absorption, indentation and fatigue resistance [7-10], smart filters [11], magnetic auxetic system [12], molecular sieves [13], seat cushions [14], superior vibration dampers [15] and acoustic isolators [16].

In the library of natural materials, $69 \%$ of the cubic elemental metals were reported to have negative Poisson's ratio (NPR) when stretched in infinitesimal strain along a specific direction [17]. Auxetic behaviour was also observed in some materials at temperature close to phase transition [18-21]. Besides, Grima et al. reported that NPR properties could be achieved by tailoring graphene [22]. Recently, the nuclei of embryonic stem cells extracted from mouse was found to be auxetic during a metastable transition state [23], which further shows the functionality of auxetic behaviour in the natural world. However, the studies of this natural auxetic behaviour are still in the preliminary stage. More detailed analysis is required for their real applications.

A variety of synthetic auxetic materials and structures were invented in the last three decades after Lakes [24] firstly presented a re-entrant foam with NPR in 1987. Wojciechowski [25] reported that Poisson's ratio can be negative in the tilted phase. Analytical solutions for both 2D [26-34] and 3D [1, 31, 35-37] auxetic materials were achieved. Because computer simulations are more powerful than just analytical method, finite element (FE) methods have been employed to investigate the auxetic materials [2, 38-41]. Limited by the fabrication techniques, few designs of 3D auxetic material were developed to the stage of practical applications [42]. One disadvantage of the most existing auxetic materials was that the auxetic behaviour only exhibited in a very limited strain range, i.e., no more than 0.1 in the design of Bückmann et al. [1]. Babaee et al. [3] extended the effective auxetic strain range to 0.3 by proposing a new design of "buckliball" as the building cell. However the geometry of their building cells was relatively complicated and the fabrication processes included extra bonding interface which resulted in the considerable scattering of the mechanical properties [2]. In addition, the base material of their models was silicone-based rubber, hence their results was limited to the elastic deformation. In contrast to elastic auxetic metamaterial, metallic auxetic metamaterial exhibits new features in their mechanical properties such as localization of plastic strain, strain hardening, and irreversible deformation. Furthermore, metallic metamaterials are usually much stronger than those made of elastomers, and therefore could carry more loads or absorb more impact energy. 
The work on metallic auxetic materials can be dated back to Lakes [43] who reported the first auxetic copper foam with non-periodic building cells in 1988. Li et al. [44] investigated the auxetic property of copper foams via resonant ultrasound spectroscopy. He et al. [45] fabricated entangled materials with a spiral wire structure which exhibited auxetic behaviour. Zhang et al. [46] reported a simple auxetic structure made of aluminium tubes and corrugated sheets. However, there was very limited research work focusing on metallic auxetic metamaterials with periodic microstructures. Only very recently, Taylor et al. [47] investigated metallic structures with NPR with 2D periodic building cells undergoing tensile deformation. Dirrenberger et al. [48] concluded that auxetic effect remained and became even more obvious with plastic yielding using finite element (FE) method but their conclusion lacked experimental support.

To check whether the design of buckling-induced elastic metamaterials can be applied to metallic based materials, we started by simply replacing the rubber based material of the auxetic metamaterials which we used in our previous work [2] with metallic material. Unexpectedly, the original auxetic behaviour exhibited in elastomer disappeared in the metallic material with identical periodic building cells during the uniaxial compression test. This finding demonstrated that the loss of auxetic behaviour in metamaterial from bucklinginduced design was related to the base material. Babaee et al. [3] successfully exploited the mechanism of buckling-induced auxetic behaviour to design a new class of 3D soft auxetic metamaterials, but the criteria they adopted could not be extended directly to metallic auxetic metamaterials.

In this paper, we conduct an in-depth investigation, both experimentally and numerically, on a new type of 3D metallic auxetic metamaterials. A series of parametric studies using validated FE models were carried out to investigate the effects of microstructures and metal plasticity on 3D metallic auxetic metamaterials.

\section{Designing the microstructures for 3D metallic auxetic metamaterials}

The methodology of generating 3D metallic auxetic metamaterials can be divided into four steps: Firstly, designing buckling-induced auxetic metamaterial; Secondly, performing buckling analysis of the original FE model with linear elastic base material; Thirdly, identifying the desirable buckling mode; Lastly, altering the geometry of the representative volume element (RVE) using the desirable buckling mode and repeating the altered RVE in three axial directions to form a 3D metallic auxetic metamaterials.

\subsection{Designing buckling-induced auxetic metamaterial}

The first step of the design approach starts with a buckling-induced auxetic metamaterial composed of a simple microstructure with regular shapes. Similar to the geometry configuration in our previous work [2], a simple cubic (SC) unit cell was generated with a diameter to edge length ratio of 1.248 which was within the limits of $1<R<\sqrt{2}$ [49]. $R$ is 
the ratio of the diameter of the sphere to the edge length of cube, as shown in figure 1(a). Figure 1(b) shows the representative volume element (RVE). The building cell was patterned in three normal directions to form the bulk metamaterial as shown in figure $1(\mathrm{c})$, where each edge of the material block was composed of eight unit cells to minimize the size effect in order to obtain reliable homogenized properties [50].

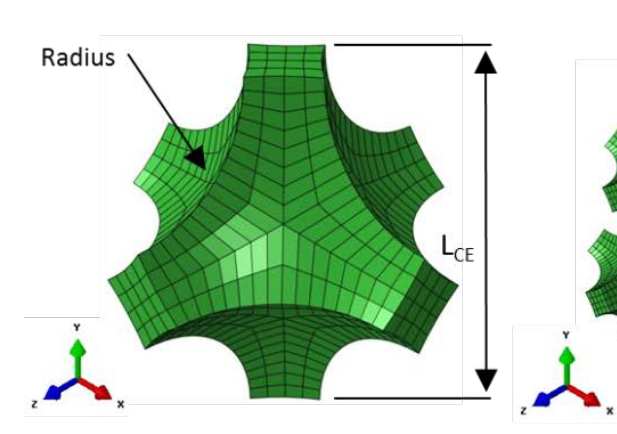

(a)

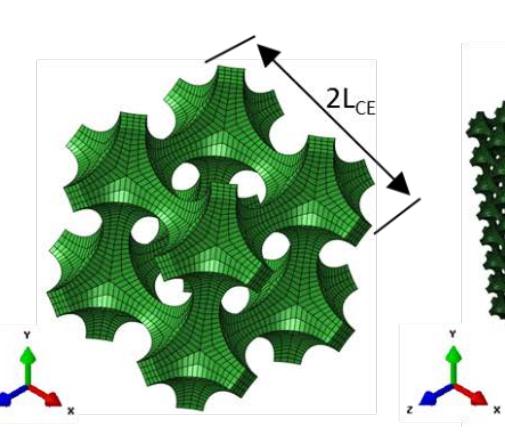

(b)

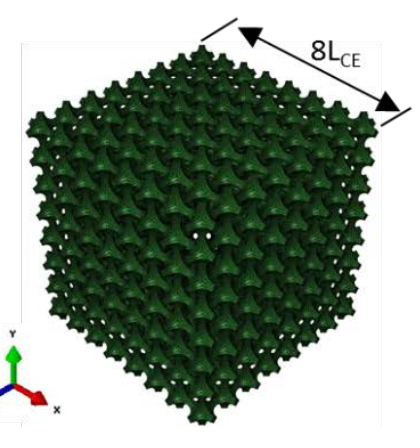

(c)

Figure 1. Initial topology design of the $3 \mathrm{D}$ buckling-induced auxetic metamaterial with cubic symmetric microstructures ( $L_{C E}=6.25 \mathrm{~mm}, R=3.9 \mathrm{~mm}$ ): (a) Building cell , (b) Representative volume element, (c) Bulk material for simulation and experiments.

\subsection{Buckling analysis of the original FE models with linear elastic base material}

The second step is to conduct buckling analysis with proper loading and boundary conditions to trigger desired buckling patterns with auxetic behaviour under uniaxial compression. The material can be any arbitrary elastomer and a modulus of $87 \mathrm{GPa}$ and Poisson's raito of 0.38 were used in our simulation. Subspace was chosen as the eigensovler during buckling analysis. The boundary condition should be carefully selected to obtain valid buckling mode. In the present work, all freedoms of nodes on the bottom and top surfaces of the FE models were constrained except for the nodal movements in the loading direction on the top surface.

A commercial finite element software package ABAQUS (Simulia, Providence, RI) was used for the buckling analysis. ABAQUS/Standard was employed for linear perturbation analysis using subspace eigensolver. The maximum number of eigenvalues of interest was set as 20 . Models were built using solid elements (ABAQUS element type C3D8 with a mesh sweeping seed size of $0.31 \mathrm{~mm}$ as shown in figure $1(\mathrm{a})$, symmetrical mesh was distributed to the FE model) and the analyses were performed under uniaxial compression.

\subsection{Identifying the desirable buckling mode}

Identifying the desired buckling pattern is based on experience from our previous and others' works $[16,47,51-55]$ where the alternating ellipsoidal pattern occurred for their auxetic materials and structures. The results of the two most desirable buckling modes both for RVE and entire model obtained from buckling analysis are presented in figure 2. RVE was picked from the central part of the entire model. 
Through buckling analysis, the first two overall buckling modes shown in figure 2(b) were found to compare well with existing literature [16, 47, 51-55], where alternating ellipsoidal pattern occurs. Interestingly, by comparing the first and second modes of the central RVE, the better symmetry feature of the second mode was observed as presented in figure 2(a) and figure 2(c). Hence the central RVE of the second pattern was selected as building cell.

\subsection{Quantifying the shape of RVE of the desirable buckling mode and patterning the altered RVE to form the bulk metamaterial}

The buckling mode is a non-dimensional infinitesimal deformation vector of the original structure. A scale factor is required to control its shape to alter the original geometrical model. Conventionally, post-buckling analysis must be turned the original bifurcation problem into a problem with continuous response with a desired deformation. This transition is accomplished by introducing a geometric imperfection pattern into the "perfect" geometry so that there is a similar deformation as that in the buckling mode. Besides, initial geometrical imperfection in a real structure is inevitable due to random errors and inaccuracy during manufacturing processes [56]. The conventional size for this imperfection is negligibly small, which will not change the overall shape of the original model. Therefore, the term "imperfection" used in our previous work [2] was unsuitable to present its true meaning as a way to change the shape of original design. Alternatively, the term of pattern scale factor (PSF) was proposed, which can determine the magnitude of buckling mode to alter the initial geometry.

In order to define PSF in our case, the central RVE of the bulk material was selected as an indicator. After the buckling analysis, the tuneable normalized buckling mode could be demonstrated by simply adding the selected mode multiplied by a deformation scale factor (DSF) to the original coordinates of all nodes. When the void in the RVE at the center of the bulk material was just closed as shown in figure 2(a), the corresponding deformation scale factor ( 0.0057 in this case) was defined as PSF $=100 \%$. Other percentages of PSF were defined accordingly such as $50 \%$ with a deformation scale factor of 0.00285 , and the $0 \%$ was the original geometry as shown in figure 2 (a).

Compared with our previous work in which we imported the overall pattern to original topology generally as shown in figure $2(\mathrm{~b})$, here the representative volume cell (RVE) at the centre of the bulk material is selected as the building cell to form the full cellular material, so the new geometry is periodic and shown in figure 3. 


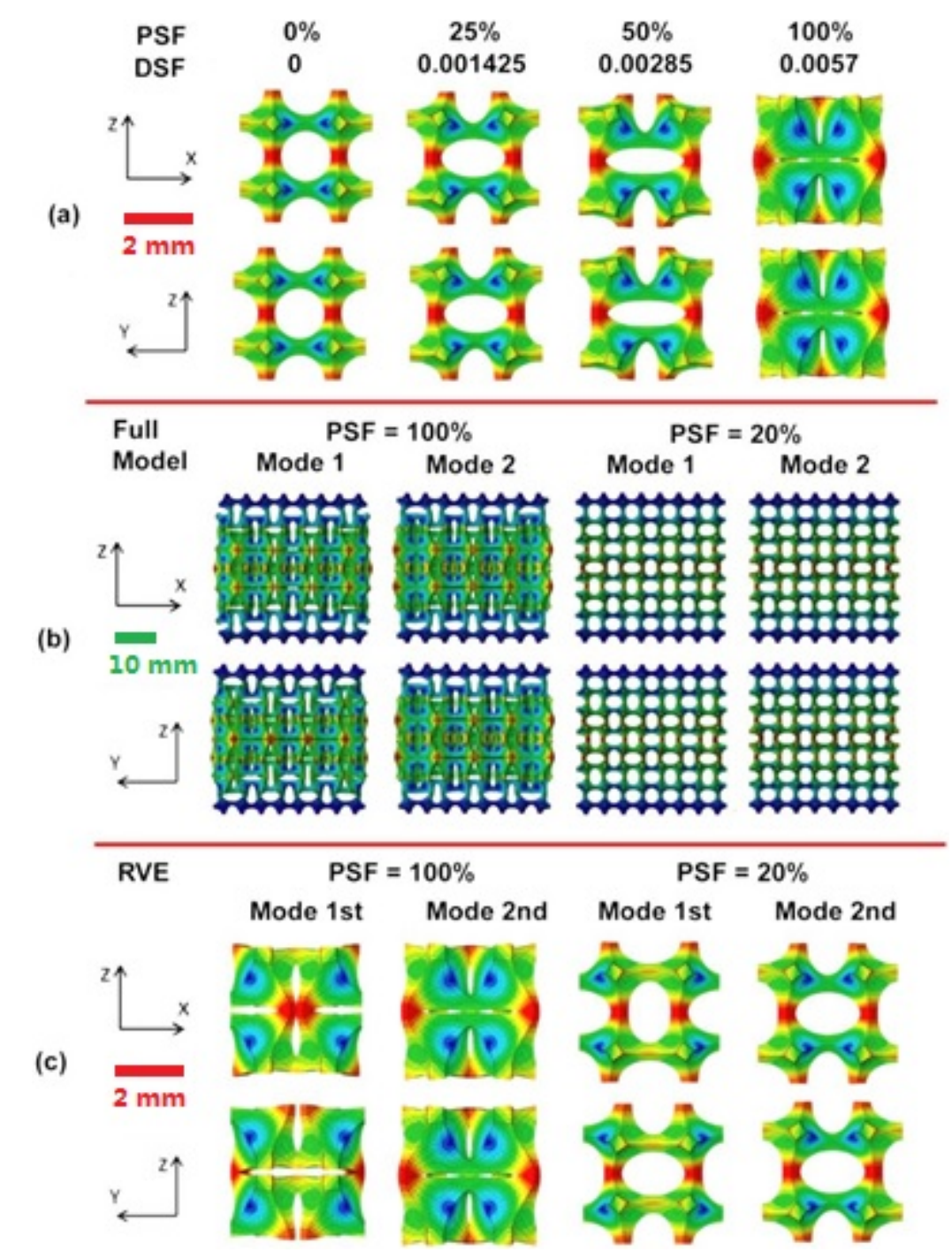

Figure 2. Two most desirable buckling modes for both RVE and entire model from two perpendicular views(Eigenvalues of the 1 st and 2 nd modes are $2.08324 \times 10^{-3}$ and $2.08473 \times 10^{-3}$ respectively.): (a) Images of the Inner-most RVE of the second mode from buckling analysis at different PSF, (b) Views of full model of the first two patterns from buckling analysis at PSF of $100 \%$ and $20 \%$, (c) Magnified views of Inter-most RVE of the first two patterns at PSF of $100 \%$ and $20 \%$. (Green scale bars: $10 \mathrm{~mm}$; Red scale bars: $2 \mathrm{~mm}$ ). 


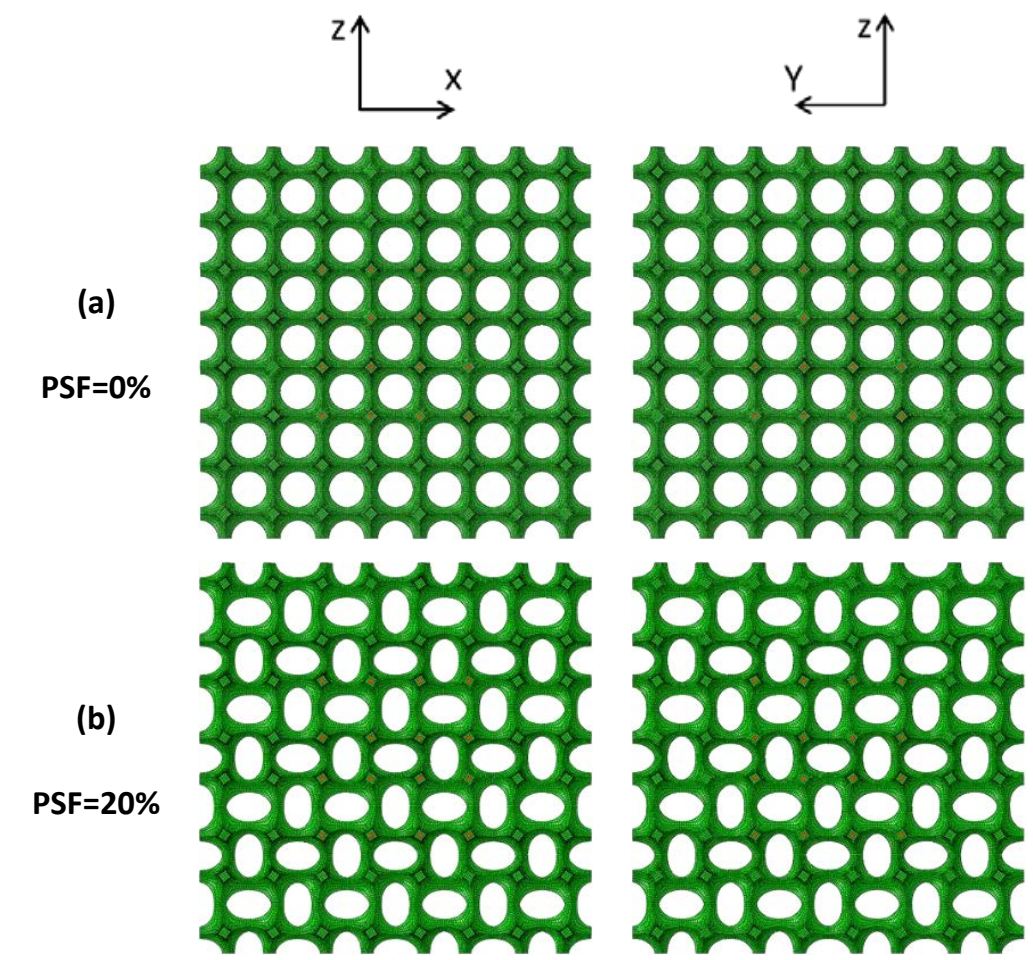

Figure 3. The side-views of two samples of the original and altered bulk metamaterials used for simulations and experimental investigations: (a) original designed buckling-induced auxetic metamaterials; (b) buckling mode altered auxetic metamaterial ideal for metallic based models.

\section{Experiment}

\subsection{Fabrication of 3D cellular metallic materials for experiments}

The specimens of the 3D cellular materials shown in figure 4 were manufactured using 3D printing (Shapeways, New York) with raw brass as the base material. It should be noted that raw brass was chosen to print our 3D cellular metamaterials mainly because of its high ductility (with an average elongation of 0.3 ).

The detailed manufacturing process of the 3D bulk metamaterials is described by Shapeways as follows: First, the model was printed in wax using a specialized high-resolution 3D printer. It was then put in a container where liquid plaster was poured in around it. Once the plaster set, the wax was melted out in a furnace, and the remaining plaster became the mould. Molten brass was poured into this mould and set to harden. The plaster was then broken away, revealing the final sample.

The overall size of the test specimens is $50 \mathrm{~mm} \times 50 \mathrm{~mm} \times 50 \mathrm{~mm}$, and the printed models are shown in figure 4 . The material properties of the printed brass base material were measured through standard tensile tests of six printed dog-bone specimens by using a MTS machine as shown in figure 5 . The results indicated that the constitutive behaviour could be accurately represented by a bilinear material model as shown in figure 6 . The average values from tensile tests of 3D printed brass dogbone are summarized in Table 1. 


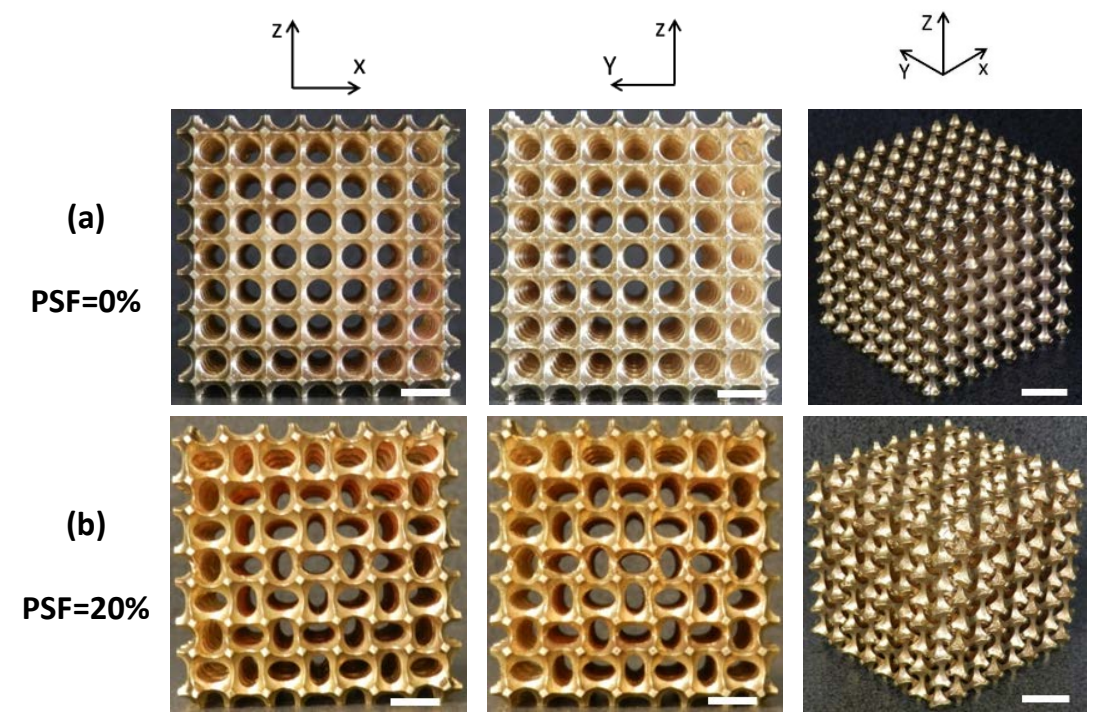

Figure 4. Two test samples for the metallic metamaterials from three different perspectives (scale bar: $10 \mathrm{~mm}$ ): (a) front view, (b) side view, (c) isometric view. (PSF=0\%: overall mass $=192.52 \mathrm{~g}$, relative density=1540.16kg/m $\mathrm{m}^{3}$, mass error=8.2\%; PSF=20\%: overall mass $=190.00 \mathrm{~g}$, relative density $=1520.00 \mathrm{~kg} / \mathrm{m}^{3}$, mass error $=6.8 \%$ ).

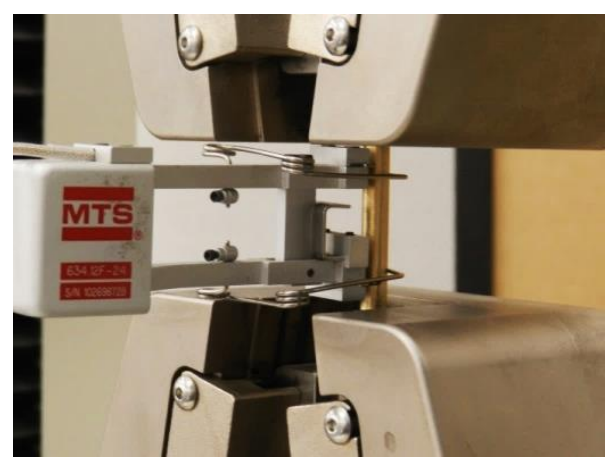

Figure 5. Test frame and extensometer arrangement for tensile test of 3D printed brass dogbone using MTS machine.

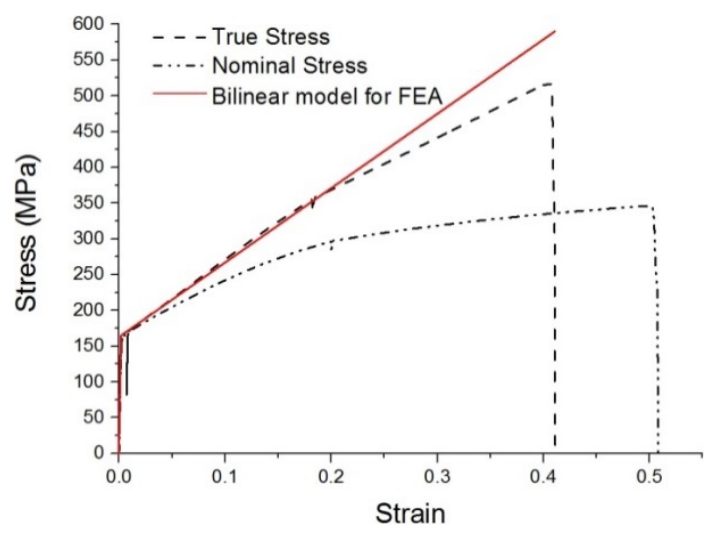

Figure 6. Illustration of true and nominal stress-strain curves for brass material produced by 3D printing and the corresponding bilinear material model for FEA.

Table 1. Table summarizing the main parameters measured from experiments of dog-bone specimens 


\begin{tabular}{l|c}
\hline & Average value \pm Error \\
\hline Young's modulus(GPa) & $86.76 \pm 20.36$ \\
Yield strength(MPa) & $140.34 \pm 89.64$ \\
Density(Kg/m $\left.{ }^{3}\right)$ & $8720 \pm 200$ \\
Strain hardening modulus(GPa) & $1.65 \pm 0.61$ \\
\hline
\end{tabular}

\subsection{Uniaxial compression tests on 3D cellular metamaterials}

The performance of our 3D auxetic metamaterials was tested using standard quasi-static uniaxial compression tests similar to those commonly used for other cellular materials [57], in which the strain rate of $10^{-3} \mathrm{~s}^{-1}$ was adopted using a Shimazu machine. A camera was used to capture the deformation in one lateral direction so as to evaluate the evolution of the Poisson's ratio of the 3D metamaterial. As the performance of the metamaterials before densification strain was our major interest, compression process was stopped manually after specimens entered densification range.

Poisson's ratio was calculated from 16 central points of ligaments at the centre region of lateral surfaces, as shown in figure 3 and figure 9. Image processing method was used for calculating Poisson's ratio of our 3D printed specimens during tests.

\subsection{New findings from experiment}

Experimental deformation processes of two metallic samples of $\mathrm{PSF}=0 \%$ and $\mathrm{PSF}=20 \%$ are demonstrated in figure 7 . The compression process of buckling-induced auxetic metamaterials indicated that the auxetic behaviour of the original geometry disappeared and was replaced with a localized deformation pattern with lateral deflection as shown in figure $7($ a). However, the auxetic behaviour remains for the metallic cellular material with $\mathrm{PSF}=20 \%$ as shown in figure $7(b)$.

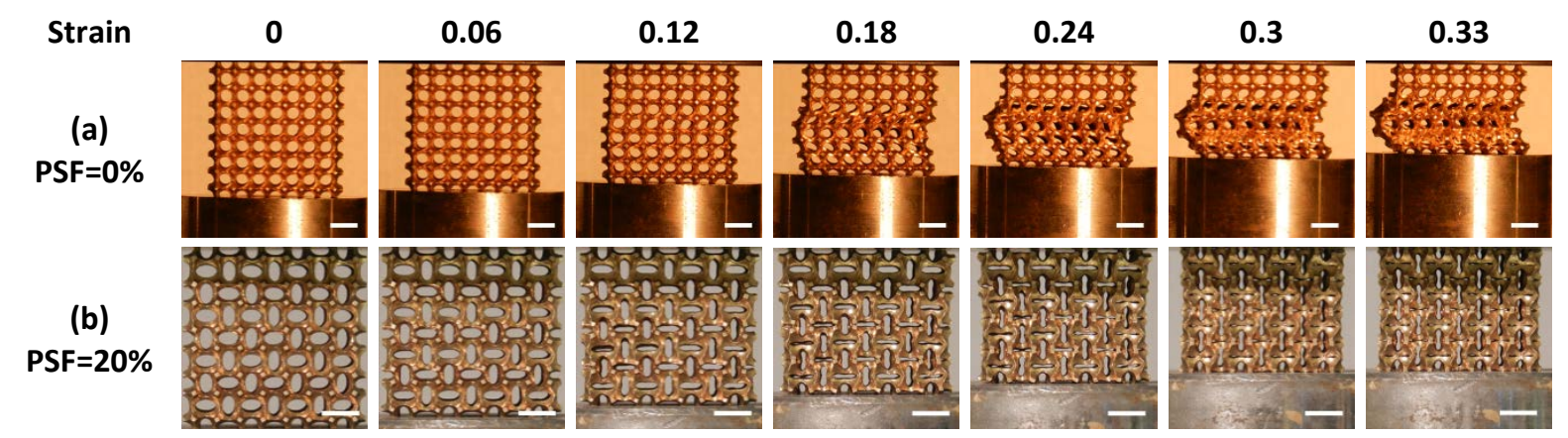

Figure 7. Images of experimental deformation processes of two metallic samples (scale bar: $10 \mathrm{~mm}$ ):

(a) Deformation processes of the metallic sample with PSF $=0 \%$, (b) Deformation processes of the metallic sample with $\mathrm{PSF}=20 \%$.

The experimental values of Poisson's ratio as a function of nominal strain are shown in figure 8. The Poisson's ratio of the original designed model was positive (very close to zero), and localized deformation pattern occurred in the test which means the initial cellular metallic metamaterial is non-auxetic. After PSF of $20 \%$ was introduced to the original geometry, the 
altered metamaterial exhibited auxetic behaviour from the very beginning as can be seen in figure 8 where the value of NPR was still decreasing when the nominal strain reached approximately 0.34 .

The deformation pattern of metallic material was compared with that of rubber material in our previous work [2]. The initial geometries of four samples before tests are shown in figure 9 from (a) to (d), and the deformed geometries of the testing specimens at the compression strain of 0.24 are illustrated in figure 9 from (e) to (h) correspondingly. It should be noted that for the original design, two different deformation patterns were observed during the experiments, auxetic deformation pattern for linear elastic based material (rubber) and localized deformation pattern with lateral deflection for metallic based material (brass).

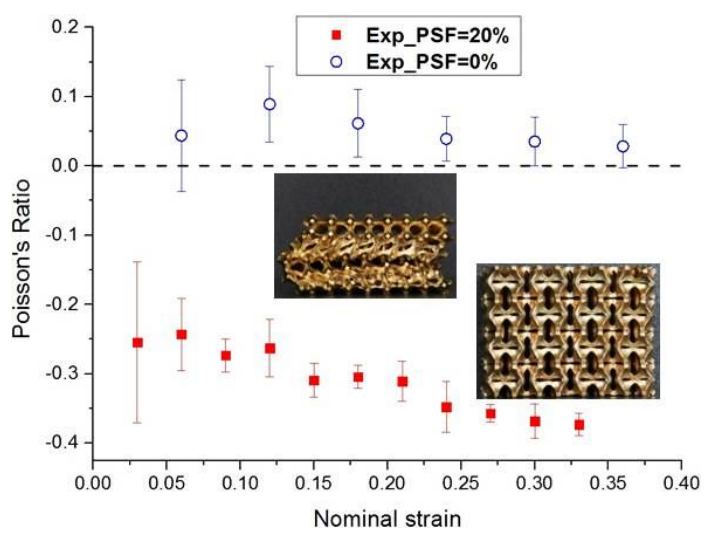

Figure 8 Numerical and experimental Poisson's ratio as a function of nominal strain for both models with $\mathrm{PSF}=0 \%$ and $\mathrm{PSF}=20 \%$, where the corresponding shape of 3D printing models after test are shown.

However, for the altered design, auxetic deformation patterns were observed both for rubber and brass samples with PSF $=20 \%$. Therefore, the loss of the auxetic behaviour in bucklinginduced auxetic metamaterials caused by the localization of plastic strain can be effectively recovered by altering the geometry of periodic RVE with a desired buckling mode as shown in figure $7(b)$ and figure $9(h)$. It is because that when the import of PSF is large enough, the deformation pattern of the buckling-induced auxetic metamaterials is settled to the prescribe pattern. Hence this confirmed the robustness of the improved methodology of generating 3D auxetic metamaterials. The experiments also indicate that a new type of 3D metallic auxetic metamaterial has been successfully generated, which is capable of undergoing large strain. 


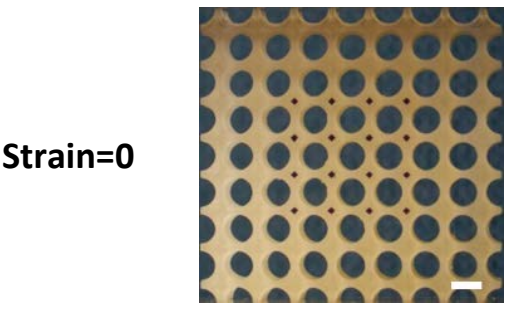

(a)

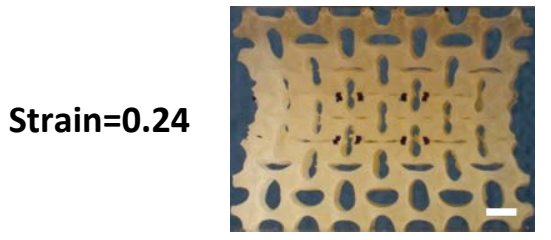

(e)

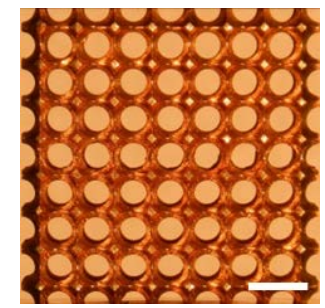

(b)

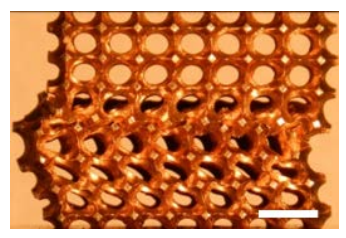

(f)

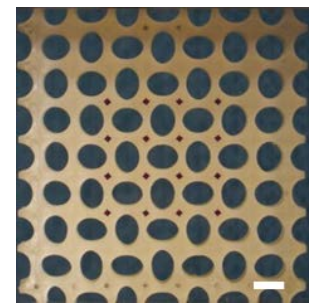

(c)

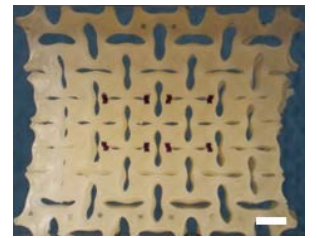

(g)

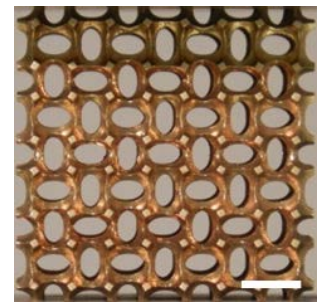

(d)

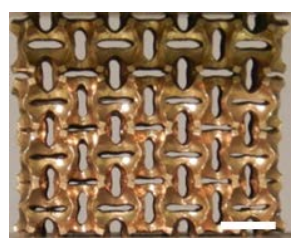

(h)

Figure 9. Experimental deformation processes for both rubber and brass samples (scale bar: $10 \mathrm{~mm}$ ): (a) Initial rubber model of original design, (b) Initial brass model of original design, (c) Initial rubber model of altered design, (d) Initial brass model of altered design, (e) Auxetic deformation pattern of rubber model after deformation for original design, ( $f$ ) Localized deformation pattern of brass model after deformation for original design, (g) Auxetic deformation pattern of rubber model after deformation for altered design, (h) Auxetic deformation pattern of brass model after deformation for altered design.

From the experimental observations, there were several new features for our metallic auxetic metamaterials. In contrast to the elastic deformation, the plastic deformation is able to dissipate energy rather than storing it in the form of internal energy [58]. Therefore, this feature can play a significant role in the fields of impact absorbing or acoustic damping related applications. In addition, the feature of irreversible deformation is necessary for some biomedical applications e.g. stents for blood vessels which require different stable patterns at different stages during the surgery operation [59]. Metallic materials can be used to fabricate negative-index metamaterials [60-62]. Besides, a magnetic field may exert its effect on the metallic auxetic metamaterials which could bring some other functional behaviour [12]. Furthermore, the plastic hardening has been used to enhance the strength of metallic materials [63], and this feature could be exploited to adjust the stiffness and strength of the metallic auxetic metamaterials.

To further explore these remarkable properties of the 3D metallic auxetic metamaterials, numerical investigations were carried out on our designed metamaterials, to examine effects of microstructures and metal plasticity.

\section{Finite element analysis}

\subsection{FE model for metallic auxetic metamaterials}

The geometries of the overall models for FE analysis are shown in figure 3. ABAQUS/Explicit solver was used for the nonlinear post-buckling analysis for taking effects of large deformations and complex self-contacts into consideration [64]. The inertia effect had been minimized by applying a gradually increased velocity on the bottom surface of the model according to Hanssen et al. [64] to guarantee zero acceleration at the beginning. Bilinear 
elastic-plastic material model was used in FE models as shown in figure 6. Young's modulus of $87 \mathrm{GPa}$ and hardening modulus of $1.7 \mathrm{GPa}$ were set for material model. The verification of mesh density and loading rate were conducted during this work.

Through the mass checking of the two printed brass models, slight difference of mass between 3D specimens and FE designed models was found that the printed models were heavier than the mass of initial design (The mass errors are $8.2 \%$ and $6.8 \%$ for the two specimens without PSF and with PSF of $20 \%$ respectively. Accordingly, we adjusted our FE model by slightly increasing the radius of cutting sphere in order to keep the mass of FE models and 3D printed models are consistent).

\subsection{FE model validation}

It is worth noting that the deformation processes of our designed 3D metallic auxetic metamaterial were identical from two different perspectives as shown in figure 10. Except for comparing the deformation processes, the FE model was also validated by comparing the stress-strain relationship with that from experiments. We have conducted the mesh dependence analysis similar to that by Pozniak et al. [65], and found the resultant Poisson's ratios from FE models with different mesh sizes are nearly identical. Thus at least four layers of elements were used for the minimal link of RVE in all our FE models.

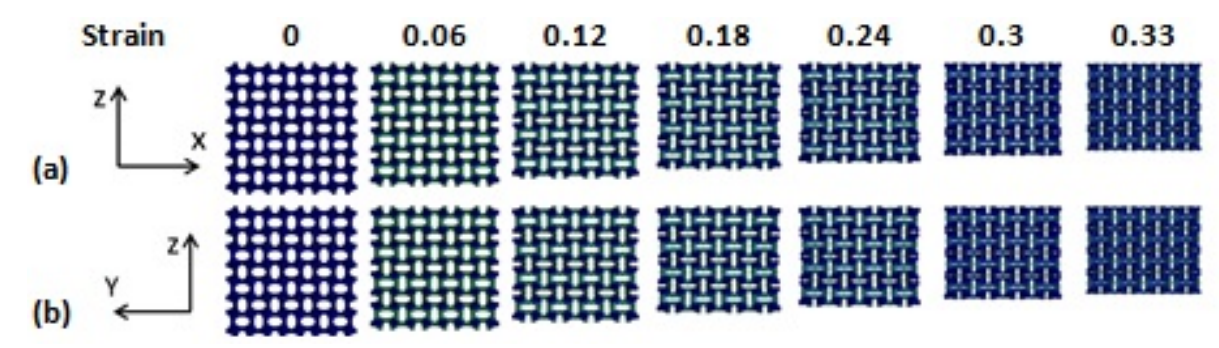

Figure 10. Deformation process of the FE model with PSF of 20\%: a) ZX-plane view, b) ZY-plane view.

The analyses were performed under uniaxial compression and the numerical results of the geometry with PSF of $20 \%$ are shown in figure 10 which compares well with the experimental result shown in figure $7(\mathrm{~b})$. The FE model with PSF of $20 \%$ was mainly validated by comparing the nominal stress-strain relationship. The comparison of these curves is shown in figure 11. However some differences still exist between experimental and FE results for the model which resulted from the irregularity of the 3D printed models in the top and bottom ends and imperfection during manufacturing process. In addition, the value of densification strain(effective strain) of the altered cellular metamaterial is marked with vertical dashed line according to [57] in figure 11, and nearly the same effective strain calculated from FE and experimental results further validates FE model. The curves obtained from numerical simulation are not very smooth, especially when it is close to the densification range as can be seen in figure 11. By carefully checking the FE field output, we confirm that those kinks were caused by the complicated contacts of the building cells. 


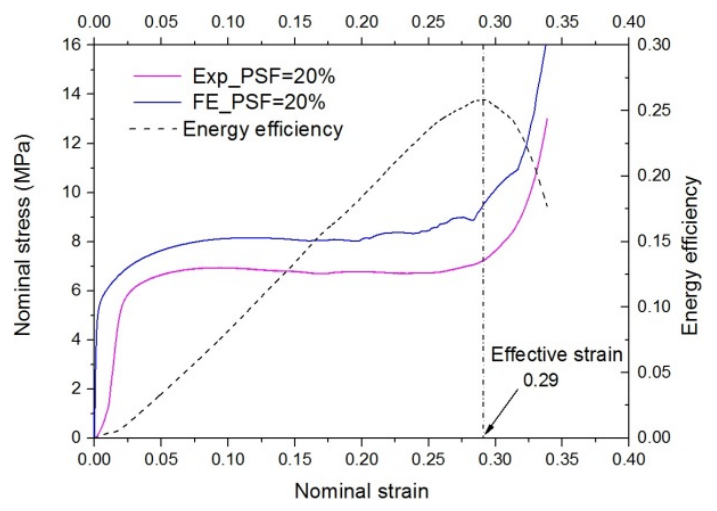

Figure 11. Comparison of nominal stress-strain curves of the improved auxetic metamaterial with PSF of $20 \%$ between experimental and FE models.

\subsection{Comparison of rubber metamaterial and brass metamaterial}

In order to examine the effect of base material on the original geometry and the availability of the proposed methodology, the comparison between the numerical and experimental results for four specimens is shown in figure 12.

It can be clearly seen from figure 12(a), original design of rubber specimen demonstrates auxetic behaviour but for the brass specimen the auxetic behaviour disappears and the plastic collapse occurs from middle layers. According to our previous work [2], the auxetic behaviour of the rubber specimen initiates from buckling. As shown in figure 12(a), although rubber specimen exhibits auxetic behaviour, the NPR behaviour does not start from the very beginning. The corresponding magnitude of Poisson's ratio still remains positive until the nominal strain reaches 0.075 . This phenomenon illustrates the loss of auxetic behaviour for the original brass model is caused by the plastic deformation occurs before the buckling of the microstructures of the brass model. In such circumstance, the buckling-induced deformation pattern cannot be triggered for the original brass model due to the sudden change of modulus of its base material. Alternatively, it crushes from the middle layers due to the localization of plastic deformation initiated by random manufacture errors.

According to the curves of Poisson's ratio as a function of nominal strain in figure 12(b), auxetic behaviour occurs from the very beginning for both of the altered rubber and altered brass models with PSF $=20 \%$. This result confirms the robustness of the proposed methodology of generating 3D auxetic metamaterials. It should be noted that the magnitudes of Poisson's ratio for altered rubber model and altered brass model are very close. This result further demonstrates the effectiveness of the proposed methodology the auxetic performance of the altered specimens are depended on their geometry rather than the chemical component of their base material. This finding agrees well with the conclusion drawn by Pozniak et al. [38], who stated that by applying harder joints one can reach lower Poisson's ratios, i.e. foams with better auxetic properties. 
Both numerical and experimental results demonstrate excellent auxetic performance of the proposed design as it is capable of sustaining auxetic behaviour over a large compression strain range up to 0.35 . A comparison indicates that our novel cellular metamaterial is superior to many existing auxetic foams as reported in [66-68] in terms of the effective strain range. Parametric studies have been performed to investigate effects of various factors on the auxetic performance and other mechanical properties, as will be discussed in later sections.

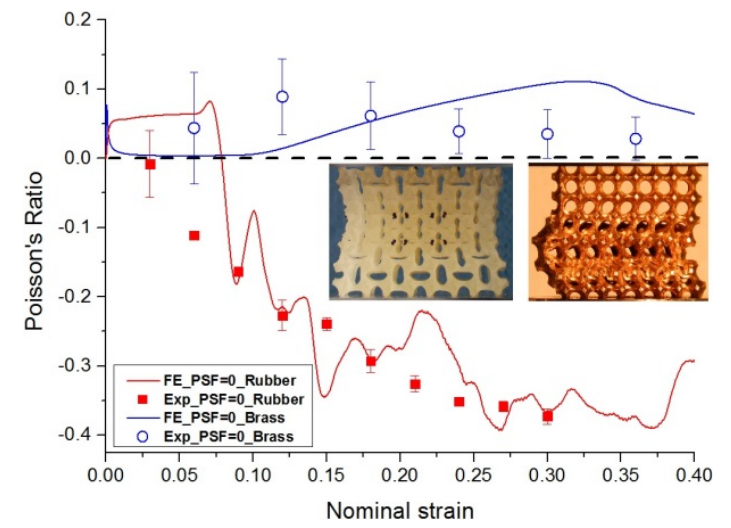

(a)

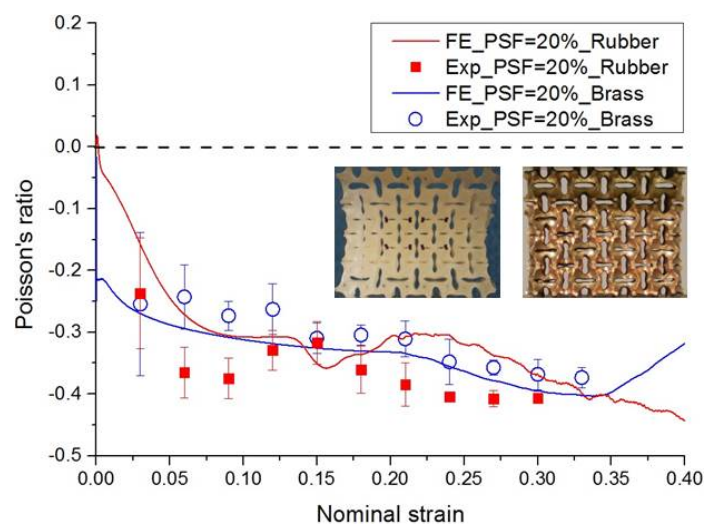

(b)

Figure 12. Curves of Poisson's ratio as a function of nominal strain: (a) curves of Poisson's ratio as a function of nominal strain for rubber and brass models with PSF=0; (b) curves of Poisson's ratio as a function of nominal strain for rubber and brass models with PSF $=20 \%$.

\subsection{Parametric studies}

\subsubsection{Effect of PSF on auxetic behaviour}

According to figure 2, the magnitude of PSF determines the shape of microstructure of our designed metallic metamaterial, which is the dominant factor for the performance of auxetic behaviour. The validated FE models were used with different values of PSF to analyse the auxetic performance in a large strain range.

The auxetic performance of the designed model can be changed through tuning the value of PSF correspondingly as shown in figure 13, where the pentagrams represent the corresponding effective strain range. The original model with $\mathrm{PSF}=0 \%$ shows non-auxetic behaviour. By increasing the value of PSF, the overall magnitude of NPR enhances while the effective strain range decreases. The reason behind this phenomenon is that when PSF is imported into the initial geometry, the original spherical voids become ellipsoidal shape which inevitably reduces effective strain range since solid material can contact easily during uniaxial compression. Therefore, the result indicates that balancing the relation between the value of NPR and effective strain range is essential before designing auxetic metallic metamaterials by using the proposed methodology of controlling the value of PSF. 


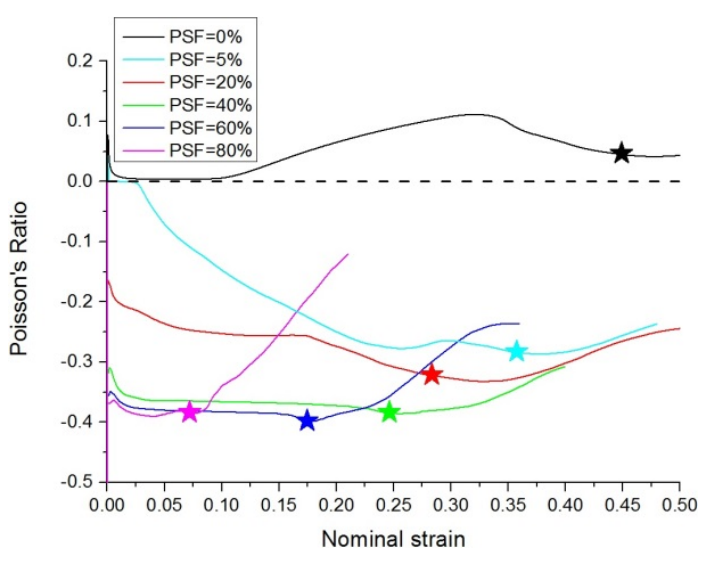

(a)

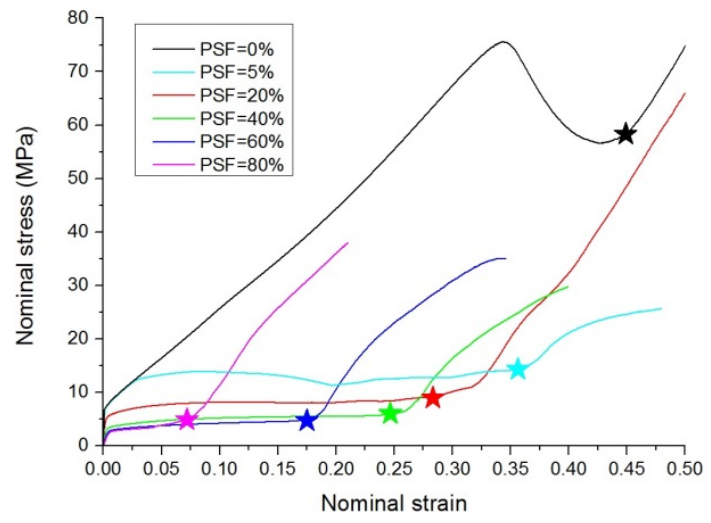

(b)

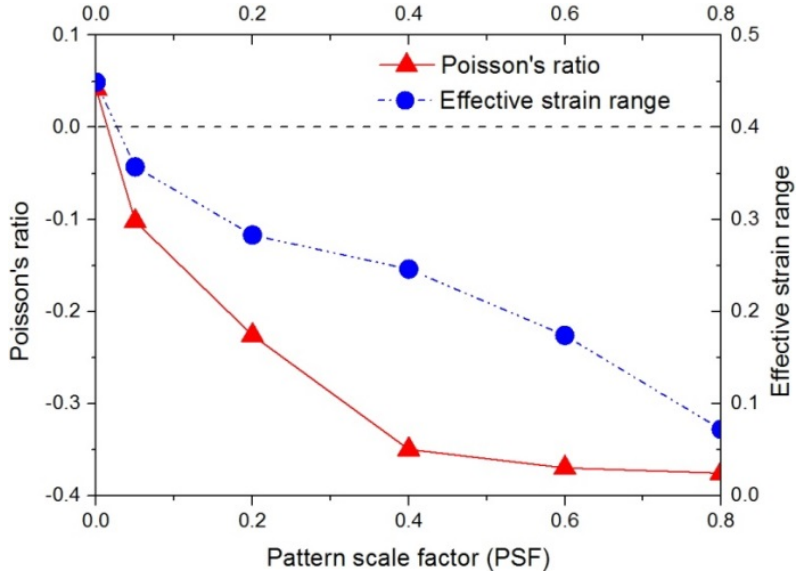

(c)

Figure 13. Effect of PSF on Poisson's ratio: (a) curves of Poisson's ratio as a function of nominal strain for models with different PSF; (b) curves of nominal stress as a function of nominal strain for models with different PSF; (c) curves of averaged Poisson's ratio and effective strain range as a function of PSF.

\subsubsection{Effect of strain hardening on auxetic behaviour}

Because of the ductility of the raw brass, the designed 3D metallic auxetic metamaterial is capable of undergoing large deformation without fracture. Under large deformation, the plastic properties of the base material of our metallic metamaterial will have significant effect on their mechanical performance. Strain hardening, as a common feature for various metals, determines the instant loading modulus of the base material. The effect of the strain hardening on the auxetic performance of our designed metallic auxetic metamaterial were investigated using the validated FE models. Bilinear elastic-plastic material model was used in FE models as shown in figure 14(c), where Es is elastic modulus and Ep is strain hardening modulus. $E p / E s=0$ means the extreme case of the true stress is stable after yield point, and $\mathrm{Ep} / \mathrm{Es}=1$ means the other extreme case where strain hardening modulus and elastic modulus are equivalent. The common value of strain hardening modulus is located between these two extreme cases.

As can be seen in figure 14(a), the differences of Poisson's ratio towards FE models with the ratio of Ep to Es from 0.2 to 1.0 are very small. That means the influence of hardening effect towards auxetic behaviour is ignorable, especially when the ratio of Ep to Es is over 0.2, the Poisson's ratio is nearly unchanged when nominal strain beyond 0.15 . Considering the 
mechanical property of our models when the ratio of Ep to Es increases from 0 to 1, the corresponding nominal stress in densification strain enhances dramatically from 4.1 MPa to 81.7 MPa.

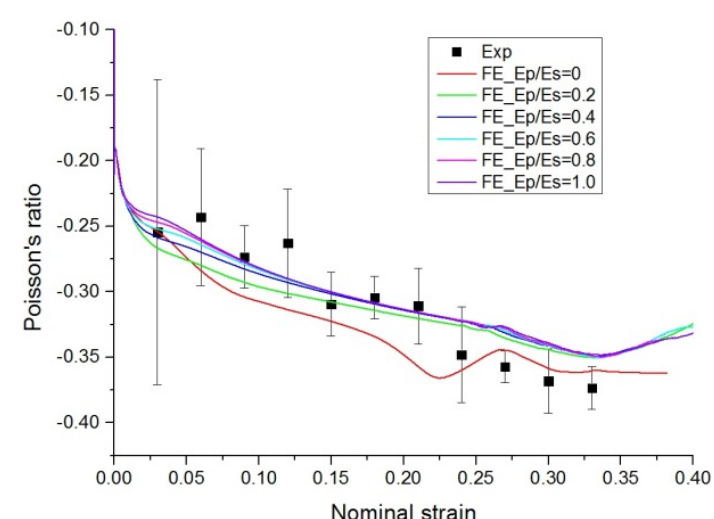

(a)

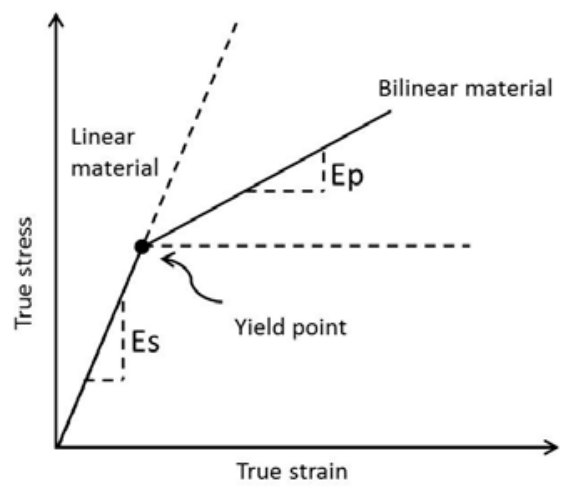

(c)

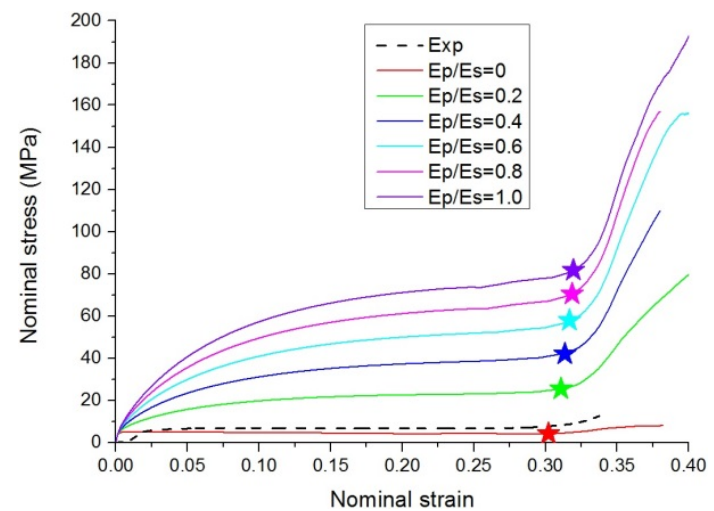

(b)

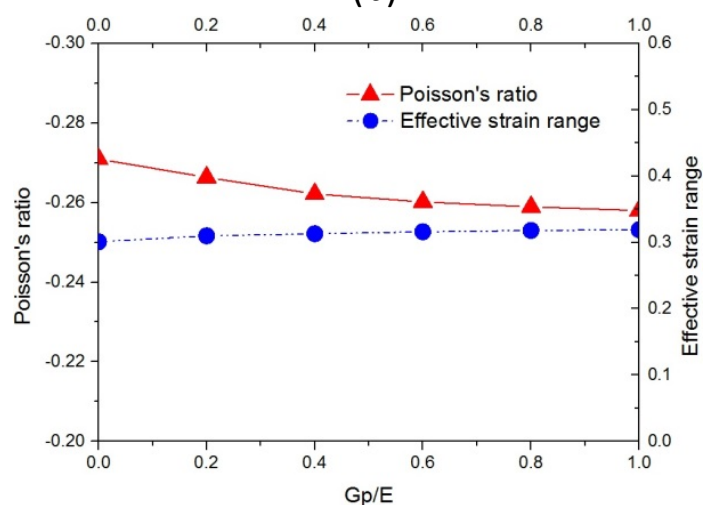

(d)

Figure 14. Parametric study of hardening effect: (a) Curves of Poisson's ratio as a function of nominal strain for models with PSF of $20 \%$ for the base materials with different values of Ep/Es. (b) Curves of nominal stress as a function of nominal strain for model with PSF of $20 \%$ for the base materials with different values of Ep/Es.

(c) Image of base material model. (d) Curves of averaged Poisson's ratio and effective strain range as a function of Ep/Es.

\subsubsection{Effect of volume fraction on auxetic behaviour}

The volume fraction influences auxetic behaviour towards the designed 3D elastic cellular metamaterials according to our previous work [2], here the parametric study of volume fraction for the designed metallic cellular metamaterials is performed. Through changing the radius of the spherical void of unit cell presented in figure 1, volume fraction can be easily adjusted correspondingly. In order to further investigate the influence of volume fraction on auxetic behaviour, the same value of PSF equals $20 \%$ was imported to the models with different radius of cutting sphere from $3.4 \mathrm{~mm}$ to $4.2 \mathrm{~mm}$. The images of RVE of FE models with different volume fraction are presented in figure 15. It should be noted that changing the value of PSF will slightly increase the volume fraction, in the present cases, the maximal volume difference between FE models with or without PSF is about $8.5 \%$. The volume fraction presented in the figure 15 is calculated from original circular hollow models. 


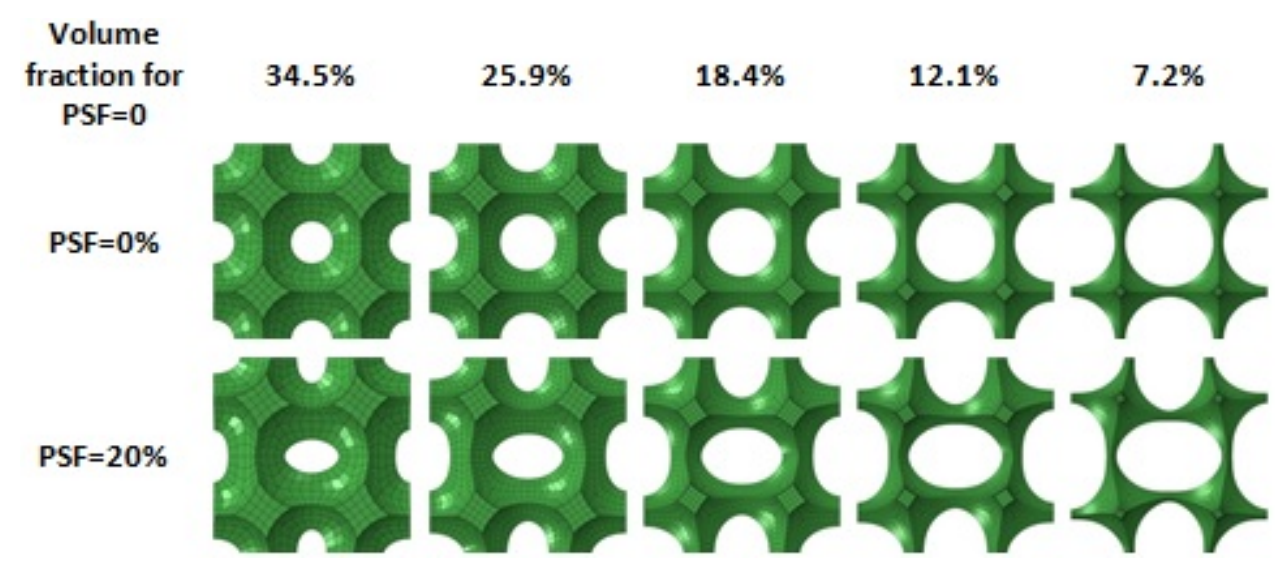

Figure 15. Representative volume elements of FE models with and without PSF at different volume fraction.

Figure 16(a) demonstrates clearly that the size of the voids has significant effect on the value of NPR. For the model with volume fraction of $34.5 \%$, the model exhibits non-auxetic behaviour. By reducing the volume fraction through the method of increasing the radius of cutting sphere, the auxetic behaviour occurs for the FE model with volume fraction of $25.9 \%$. Hence the critical volume fraction for our model lies in the values between $25.9 \%$ and $34.5 \%$.

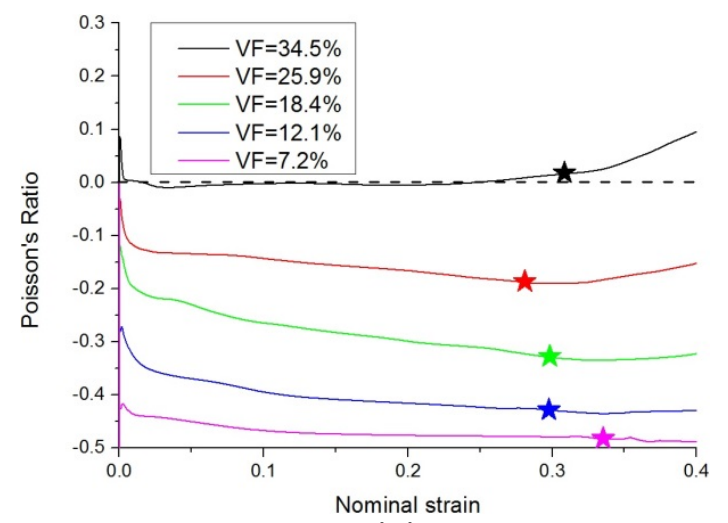

(a)

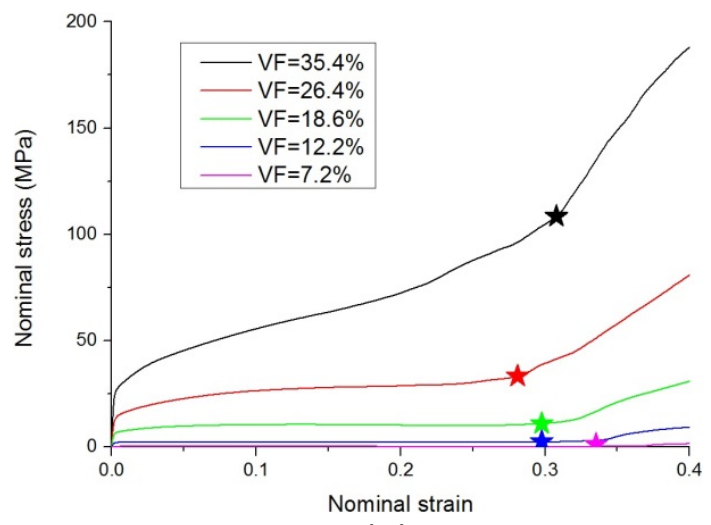

(b)

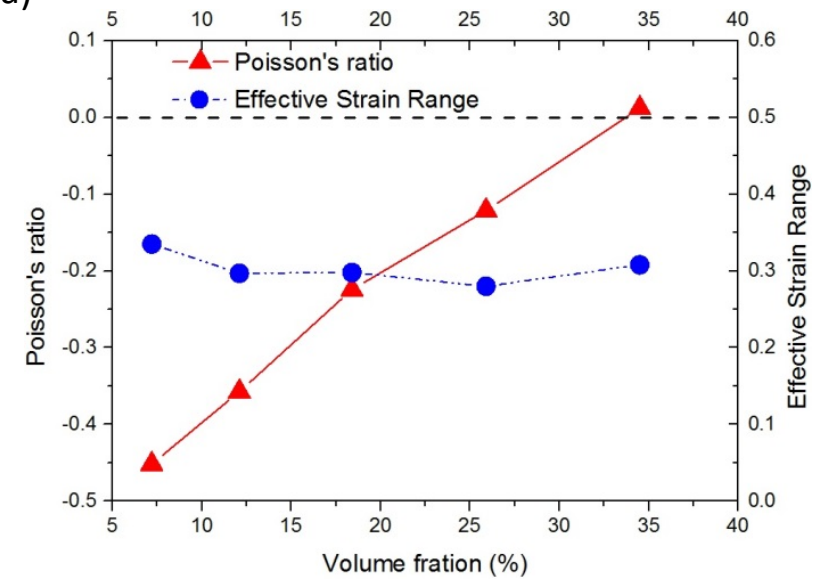

(c)

Figure 16 Effect of volume fraction on Poisson's ratio: (a) curves of Poisson's ratio as a function of nominal strain towards models in different volume fraction with PSF of 20\%; (b) Curves of nominal stress as a function of nominal strain towards model in different volume fraction with PSF of 20\%; (c) Curves of averaged Poisson's ratio and effective strain range as a function of volume fraction. 
And the auxetic performance can be enhanced by reducing volume fraction. The effective strain range of the models in the given volume fraction remains stable around 0.3. From figure $16(b)$, the mechanical property of our models is very sensitive to volume fraction. When volume fraction of our models reduces from $34.5 \%$ to $7.2 \%$, the corresponding nominal stress in densification strain drops dramatically from $107 \mathrm{MPa}$ to nearly $0.25 \mathrm{MPa}$.

\section{Concluding remarks}

The auxetic performance of a newly invented 3D metallic metamaterial was investigated experimentally and numerically in this paper. Inspired by the loss of auxetic behaviour of buckling-induced auxetic metamaterials with a metallic base material, a new methodology was developed and its effectiveness was proved experimentally and numerically. The effects of several key parameters on the auxetic performance and mechanical properties were explored using validated finite element models. Through these investigations, the following conclusions can be drawn:

1) The buckling-induced auxetic metamaterial may lose its auxetic behaviour when the base material is changed from an elastomer to a ductile metal.

2) The method of generating 3D metallic auxetic metamaterial by introducing PSF has been proposed and its effectiveness has been validated through both numerical and experimental methods.

3) The loss of NPR behaviour in the previous auxetic metamaterial design is attributed to the localization of plastic deformation of the metallic base material.

4) Plastic strain hardening has a negligible effect on auxetic behaviour of the designed 3D metallic auxetic metamaterial.

5) The auxetic performance of our 3D metallic metamaterial can be controlled by PSF and the volume fraction.

Based on these novel features, a wide range of potential applications of the designed 3D metallic auxetic metamaterial are anticipated because of their large effective strain range, higher stiffness and strength than elastomer based metamaterials, periodic microstructure and simple tuning property by using one single control parameter of PSF. The advantages mentioned above make the designed metallic metamaterial superior to the most conventional auxetic foam and other non-periodic auxetic materials in these aspects. It is worth noting that the newly designed 3D metallic metamaterials will exhibit auxetic behaviour not only in compression but also in tension. Based on the novelty of the methodology and superior properties of our designed 3D auxetic metallic metamaterials in this work, it is expected that the developed methodology proposed here will inspire other researchers to generate more 3D metallic auxetic metamaterials. It should be pointed out that there are several limitations for the completeness of the methodology presented in the paper. The desired buckling mode with auxetic behaviour may not be suitable for all periodic cellular materials. An effective way to identify the desirable buckling pattern is required to facilitate the search for new types of auxetic structures. The metamaterial designed in the 
current paper is anisotropic (cubic symmetric) and more symmetric constraints should applied to obtain anisotropic metamaterial. In the 3D auxetic metallic metamaterials, the value of NPR is not constant over its effective strain range, which may limit their practical applications. Therefore, topology optimization work aimed at this requirement is necessary in future research.

\section{Acknowledgments}

This work was supported by the Australian Research Council (DP140100213), the China Scholarship Council (201306370057), the Major Program of the National Natural Science Foundation of China (U1334208), and the Postgraduate Innovation Foundation of Central South University (2013zzts040).

\section{References}

[1] T. Bückmann, N. Stenger, M. Kadic, J. Kaschke, A. Frölich, T. Kennerknecht, C. Eberl, M. Thiel, M. Wegener, Advanced Materials, 24 (2012) 2710-2714.

[2] J. Shen, S. Zhou, X. Huang, Y.M. Xie, Physica Status Solidi B, 251 (2014) 1515-1522.

[3] S. Babaee, J. Shim, J.C. Weaver, E.R. Chen, N. Patel, K. Bertoldi, Advanced Materials, 25 (2013) 5044-5049.

[4] K.E. Evans, M.A. Nkansah, I.J. Hutchinson, S.C. Rogers, Nature, 353 (1991) 124-124.

[5] F. Scarpa, Signal Processing Magazine, IEEE, 25 (2008) 128-126.

[6] B. Xu, F. Arias, S.T. Brittain, X.M. Zhao, B. Grzybowski, S. Torquato, G.M. Whitesides, Advanced Materials, 11 (1999) 1186-1189.

[7] K.L. Alderson, V.R. Simkins, V.L. Coenen, P.J. Davies, A. Alderson, K.E. Evans, Physica Status Solidi B, 242 (2005) 509-518.

[8] A. Bezazi, F. Scarpa, International Journal of Fatigue, 29 (2007) 922-930.

[9] A. Bezazi, F. Scarpa, International Journal of Fatigue, 31 (2009) 488-494.

[10] K.E. Evans, A. Alderson, Advanced Materials, 12 (2000) 617-628.

[11] A. Alderson, J. Rasburn, S. Ameer-Beg, P.G. Mullarkey, W. Perrie, K.E. Evans, Industrial \& Engineering Chemistry Research, 39 (2000) 654-665.

[12] J.N. Grima, R. Caruana-Gauchi, M.R.Dudek, K.W.Wojciechowski, R. Gatt, Smart Materials and Structures, 22 (2013) 084016 
[13] J.N. Grima, R. Jackson, A. Alderson, K.E. Evans, Advanced Materials, 12 (2000) 19121918.

[14] Y.-C. Wang, R. Lakes, International Journal of Solids and Structures, 39 (2002) 4825-4838.

[15] M. Yanhong, S. Fabrizio, Z. Dayi, Z. Bin, C. Lulu, H. Jie, Smart Materials and Structures, 22 (2013) 084012.

[16] K. Bertoldi, P.M. Reis, S. Willshaw, T. Mullin, Advanced Materials, 22 (2010) 361-366.

[17] R.H. Baughman, J.M. Shacklette, A.A. Zakhidov, S. Stafstrom, Nature, 392 (1998) 362365.

[18] S. Hirotsu, Macromolecules, 23 (1990) 903-905.

[19] S. Hirotsu, Journal of Chemical Physics, 94 (1991) 3949-3957.

[20] C. Li, Z. Hu, Y. Li, Physical Review E, 48 (1993) 603-606.

[21] E.A.M. Ruth, T. Moxon, A. Buckley, P.A. Taylor, T.W. Darling, M.A. Carpenter, Journal of Physics: Condensed Matter, 20 (2008) 075229.

[22] J.N. Grima, S. Winczewski, L. Mizzi, M.C. Grech, R. Cauchi, R. Gatt, D. Attard, K.W. Wojciechowski, J. Rybicki, Advanced Materials, 27 (2015) 1455-1459.

[23] K.F. Stefano Pagliara, Crystal R. McClain, George W. Wylde, Cynthia L. Fisher, Nature Materials, 13 (2014) 638-644.

[24] R. Lakes, Science, 235 (1987) 1038-1040.

[25] K.W. Wojciechowski, Molecular Physics, 61 (1987) 1247-1258.

[26] J. N. Grima, A. Alderson, and K. E. Evans, Physica Status Solidi B, 242 (2005) 561-575.

[27] A. Alderson, K.L. Alderson, K.E. Evans, J.N. Grima, M.R. Williams, P.J. Davies, Physica Status Solidi B, 242 (2005) 499-508.

[28] D. Attard, E. Manicaro, R. Gatt, J.N. Grima, Physica Status Solidi B, 246 (2009) 2045-2054.

[29] E. Chetcuti, B. Ellul, E. Manicaro, J.-P. Brincat, D. Attard, R. Gatt, J.N. Grima, Physica Status Solidi B, 251 (2014) 297-306.

[30] L. Teik-Cheng, Smart Materials and Structures, 23 (2014) 045004.

[31] R.F. Almgren, Journal of Elasticity, 15 (1985) 427-430.

[32] K.W. Wojciechowski, Physics Letters A, 137 (1989) 60-64. 
[33] G. Wei, S.F. Edwards, Physica A: Statistical Mechanics and its Applications, 258 (1998) 5-10.

[34] K.W. Wojciechowski, Journal of Physics A: Mathematical and General, 36 (2003) 11765.

[35] T.P. Hughes, A. Marmier, K.E. Evans, International Journal of Solids and Structures, 47 (2010) 1469-1476.

[36] P.V. Pikhitsa, M. Choi, H.-J. Kim, S.-H. Ahn, Physica Status Solidi B, 246 (2009) 20982101.

[37] K. Nicholas, B. Gaetano, Smart Materials and Structures, 22 (2013) 084008.

[38] A.A. Pozniak, J. Smardzewski, K.W. Wojciechowski, Smart Materials and Structures, 22 (2013) 084009.

[39] S.A. McDonald, G. Dedreuil-Monet, Y.T. Yao, A. Alderson, P.J. Withers, Physica Status Solidi B, 248 (2011) 45-51.

[40] T. Strek, B. Maruszewski, J.W. Narojczyk, K.W. Wojciechowski, Journal of NonCrystalline Solids, 354 (2008) 4475-4480.

[41] T. Strek, P. Kedziora, B. Maruszewski, A.A. Pozniak, K.V. Tretiakov, K.W. Wojciechowski, Journal of Non-Crystalline Solids, 355 (2009) 1387-1392.

[42] J.-H. Lee, J.P. Singer, E.L. Thomas, Advanced Materials, 24 (2012) 4782-4810.

[43] E.A. Friis, R.S. Lakes, J.B. Park, Journal of Materials Science, 23 (1988) 4406-4414.

[44] D. Li, L. Dong, R.S. Lakes, Physica Status Solidi B, 250 (2013) 1983-1987.

[45] G. He, Q. Tan, G. Jiang, Q. Li, Smart Materials and Structures, 23 (2014) 095011.

[46] Z. Zhang, H. Hu, S. Liu, B. Xu, Physica Status Solidi B, 250 (2013) 1996-2001.

[47] M. Taylor, L. Francesconi, M. Gerendás, A. Shanian, C. Carson, K. Bertoldi, Advanced Materials, 26 (2014) 2365-2370.

[48] J. Dirrenberger, S. Forest, D. Jeulin, Computational Materials Science, 64 (2012) 57-61.

[49] M. Maldovan, C.K. Ullal, J.H. Jang, E.L. Thomas, Advanced Materials, 19 (2007) 38093813.

[50] M.F. Ashby, A.G. Evans, N.A. Fleck, L.J. Gibson, J.W. Hutchinson, H.N.G. Wadley, Chapter 3 - Characterization methods, in: M.F. Ashby, A.G. Evans, N.A. Fleck, L.J. Gibson, J.W. Hutchinson, H.N.G. Wadley (Eds.) Metal Foams, Butterworth-Heinemann, Burlington, 2000, pp. 24-39. 
[51] T. Mullin, S. Willshaw, F. Box, Soft Matter, 9 (2013) 4951-4955.

[52] J.T.B. Overvelde, K. Bertoldi, Journal of the Mechanics and Physics of Solids, 64 (2014) 351-366.

[53] J.T.B. Overvelde, S. Shan, K. Bertoldi, Advanced Materials, 24 (2012) 2337-2342.

[54] P. Wang, J. Shim, K. Bertoldi, Physical Review B, 88 (2013) 014304.

[55] S. Willshaw, T. Mullin, Soft Matter, 8 (2012) 1747-1750.

[56] I. Elishakoff, G.Q. Cai, J.H. Starnes Jr, International Journal of Non-Linear Mechanics, 29 (1994) 71-82.

[57] J. Shen, Y. Min Xie, X. Huang, S. Zhou, D. Ruan, Journal of the Mechanical Behavior of Biomedical Materials, 15 (2012) 141-152.

[58] G. Lu, T.X. Yu, Energy Absorption of Structures and Materials, Woodhead Publishing Ltd, Cambridge, 2003.

[59] R. Gatt, R. Caruana-Gauci, D. Attard, A.R. Casha, W. Wolak, K. Dudek, L. Mizzi, J.N. Grima, Physica Status Solidi B, 2 (2014) 321-327.

[60] F. Fang, Y. Cheng, H. Liao, Physica Scripta, 89 (2014) 025501.

[61] Y.Z. Cheng, Y. Nie, R.Z. Gong, European Physical Journal B, 85 (2012) 1-6.

[62] X.-J. He, Y. Wang, Z.-X. Geng, J.-M. Wang, T.-I. Gui, Journal of Magnetism and Magnetic Materials, 323 (2011) 2425-2428.

[63] J.L. Chaboche, International Journal of Plasticity, 24 (2008) 1642-1693.

[64] A.G. Hanssen, O.S. Hopperstad, M. Langseth, H. Ilstad, International Journal of Mechanical Sciences, 44 (2002) 359-406.

[65] A.A. Pozniak, K.W. Wojciechowski, Physica Status Solidi B, 251 (2014) 367-374.

[66] K. Alderson, A. Alderson, N. Ravirala, V. Simkins, P. Davies, Physica Status Solidi B, 249 (2012) 1315-1321.

[67] A. Andersson, S. Lundmark, A. Magnusson, F.H.J. Maurer, Journal of Applied Polymer Science, 111 (2009) 2290-2298.

[68] P. Pastorino, F. Scarpa, S. Patsias, J.R. Yates, S.J. Haake, M. Ruzzene, Physica Status Solidi B, 244 (2007) 955-965. 